Service social

\title{
Jeunesses et pratiques sociales : à l'ombre des résistances, un questionnement inévitable
}

\section{Jean-François René}

Volume 35, numéro 3, 1986

Les jeunes et le travail social

URI : https://id.erudit.org/iderudit/706313ar

DOI : https://doi.org/10.7202/706313ar

Aller au sommaire du numéro

Éditeur(s)

École de service social de l'Université Laval

ISSN

1708-1734 (numérique)

Découvrir la revue

Citer cet article

René, J.-F. (1986). Jeunesses et pratiques sociales : à l'ombre des résistances, un questionnement inévitable. Service social, 35(3), 294-303.

https://doi.org/10.7202/706313ar
Résumé de l'article

Dans cet article, est remis en question le constat d'individualisme chez les jeunes d'aujourd'hui. Plutôt conservateurs, d'un conservatisme qui comporte néanmoins une « intéressante vitalité », les jeunes développent une sociabilité de réseau nouvelle.

Transposée dans le cadre des débats sur la privatisation des services sociaux, cette sociabilité s'exprime, soit par la déviance sociale, soit par le recours à des réseaux de nature substitutive, non institutionnels et plus communautaires. 


\section{Jeunesses et pratiques sociales : à l’ombre des résistances, un questionnement inévitable}

\section{Jean-François René}

Cet article a pour but de mettre en relief quelques traits dominants de la jeunesse d'aujourd'hui, de les mettre en relation et d'en tirer les conséquences pour la pratique sociale.

\section{Une jeunesse individualiste?}

Même si nous voulions le nier, il n'est pas possible de passer sous silence le fait qu'il y a présentement persistance et même accentuation des tendances individualistes au sein des valeurs de la (des) jeunesse(s) québécoise(s).

À cause de la crise économique, c'est l'ère du chacun pour soi et de la course à la performance, seules avenues possibles pour s'assurer une hypothétique place au soleil. Chacun semble centré sur ses études, sa recherche de travail, son avenir, etc. C'est un peu le "Sauve qui peut, sa vie " qui prime, en réponse à une société qui privilégie, conjoncturellement, mais aussi en termes d'évolution socio-historique, un tel état de fait.

"Si l'individualisme gagne du terrain aux dépens de l'action collective, la motivation pour les études y est peut-être pour quelque chose. [...] les jeunes qui, à défaut d'autres perspectives, poursuivent des études, viennent d'abord à l'école pour se donner de meilleures chances de réussir socialement. Le diplôme est perçu comme un investissement à risque permettant de se prémunir contre le chômage. " 1

Cette nécessité de performer pour survivre, pour " ouvrir " l'avenir, s'accompagne d'une autre tendance qu'il faut lire, à la fois en lien avec 
cet individualisme compétitif, mais aussi différemment. Pensons ici à cette course à "son bien-être", à "son intimité ", à "ses besoins", à ce développement de l'amour propre et du narcissisme, si caractéristique de l'homme contemporain et de la jeune génération.

\begin{abstract}
" lls cherchent à être bien, malgré tout. Ils cherchent à être bien tout de suite, ne voulant sacrifier leur vie sur aucun autel [...] les jeunes comme les moins jeunes se réfugient où ils peuvent. On se retrouve tous dans une même recherche du bien-être immédiat [...] dans ses histoires d'amour [...] dans son corps, par le jogging, la bonne alimentation, ou le aki! " 2
\end{abstract}

Face à cette réalilté, nous sommes en droit de nous demander si les Bell, Lasch, Lipovetsky et autres n'ont pas en partie raison d'affirmer que nous sommes maintenant rendus à l'ère de l'homo psychologicus, que l'individualisme hédoniste l'emporte ou, tout au moins, partage le terrain avec ce vieil individualisme compétitif et conquérant, jadis si caractéristique d'une certaine Amérique.

Il nous faut répondre de manière évasive et ambiguë à cette question. S'il y a définitivement présence d'un certain narcissisme dans le vécu de la jeunesse occidentale, on ne peut adhérer véritablement à ce courant analytique. Cette course au bien-être personnel n'est pas exclusivement narcissique et peut être lue, a contrario, comme un désir plus ou moins conscient de retrouver des valeurs collectives, perdues dans les méandres de la conquête et de l'esprit de compétition.

\title{
Et le conservatisme?
}

Peut-on aussi parler d'une jeunesse plutôt conservatrice ? En fait, il y a des traits, des données qui semblent démontrer un certain conservatisme chez bien des jeunes d'aujourd'hui, entre autres par rapport à certaines tentatives de transformation sociale qui marquèrent les années 1960 et 1970. C'est particulièrement le cas de la conception des rapports hommes-femmes que véhiculent les plus jeunes de ces jeunesses (au sens de génération, ce qui n'est pas un découpage exclusif), soit les 15-19 ans. Prenons ici à témoin une étude récente du Conseil consultatif canadien sur la situation de la femme (C.C.C.S.F.), qui souligne que seulement $25 \%$ des jeunes filles interrogées se déclarent en faveur du partage des tâches ménagères, et que $45 \%$ font du mariage l'objectif central de leur vie. ${ }^{3}$

Dans le même sens, une enquête menée récemment dans un cégep de la rive-sud de Montréal auprès de 500 jeunes et portant sur les rapports amoureux, révèle que $96 \%$ des jeunes interrogés jugent la 
fidélité essentielle dans une relation de couple, et que $62 \%$ tiennent à vivre leur vie sexuelle à l'intérieur d'un lien amoureux . ${ }^{4} \mathrm{Un}$ article sur ce sondage, rédigé dans $L a$ Presse du $1^{\text {er }}$ mars 1986, rappelle qu'il y a dix ans, une autre enquête donnait des résultats assez différents, lire : moins "conservateurs", plus proches de la libération sexuelle de la fin des années 1960.

Qu'est-ce à dire? Que nous pourrions multiplier les exemples? Sûrement ! Que nous pourrions nuancer? Aussi ! En ce sens, l'étiquette "plus conservateur " n'est pas dénuée de fondement mais demande un usage prudent. II n'est pas certain, loin de là, que ce soit valable pour tout, et il est essentiel de dépasser ce genre de constat facile et fort superficiel. Si l'époque des emballements de toute nature, autant amoureux que politiques, est révolue, il faut éviter de qualifier de conservatisme criant ce qui est peut-être porteur, en partie, d'autre chose.

\section{S'organiser et résister autrement}

Cette "autre chose" constitue le thème dominant d'un article portant sur les "résistances" qui apparaissent et se développent de multiples façons, et qui mettent à jour des formes d'agrégation souvent fort différentes de ce que nous pouvions observer il y a seulement cinq ou dix ans . ${ }^{5}$ Rappelons-en, ici, les principaux éléments, essentiellement parce qu'ils touchent et rendent plus compréhensibles les constats sur les notions d'individualisme et de conservatisme traitées plus tôt.

Ainsi, nous pouvons actuellement constater, dans la jeunesse québécoise, le développement d'une sociabilité de réseau, plus ou moins formelle, plus ou moins organisée, autour de laquelle gravitent amis et, parfois, famille élargie : réseaux d'entraide, souvent peu structurés, lieux où s'affirment d'abord et avant tout des solidarités nouvelles qui se substituent aux solidarités mécaniques propres au social-étatique, et qui s'expriment souvent par le biais d'un art consommé de la débrouillardise sociale. Ces réseaux peuvent comporter des éléments foncièrement transgressifs: transgression des codes, des normes, des lois sociales, dans un but évident, mais souvent inconscient, d'assurer et de perpétuer la survie et la qualité de vie, tant des individus que desdits réseaux.

En voici un exemple fort explicite : une demi-douzaine de jeunes adultes vivent ensemble, partageant entre eux deux logements d'un même immeuble. Ils vivent tous de prestations du bien-être social. Chaque membre du groupe a développé une " compétence » dans l'art de débrouillage transgressif. Ainsi, l'un des membres du réseau est pusher; il alimente le groupe en drogue mais il ramène aussi l'argent de 
ses ventes ce qui permet d'arrondir les fins de mois. Un autre est un expert du vol à l'étalage. Il se fait donc un devoir de fournir à tous les membres du groupe chemises, pantalons et évidemment de la bonne taille! Un troisième est devenu un expert dans l'art de falsifier des documents légaux, expertise non négligeable lorsqu'on connaît l'ampleur du fouillis administratif auquel doivent faire face les assistés sociaux.

Ces réseaux peuvent aussi s'exprimer dans un univers où la résistance à l'ordre établi se fait plus " alternative ", plus créatrice. C'est particulièrement le cas des jeunes qui désirent créer leur emploi, parfois dans des cadres communautaires, parfois plus près de l'entrepreneurship traditionnel. L'explosion récente, depuis environ cinq ans, de toute une série de pratiques communautaires du type "Maison de jeunes", démontre ce désir de travail autonome (au sens du fonctionnement), en lien avec le milieu et au service de ce milieu, et ce malgré les limites évidentes qu'impose un certain encadrement étatique. Globalement, ces constats font surgir une vie très ancrée dans le quotidien, une vie qui secrète des solidarités de base, immédiates, organiques, pas nécessairement alternatives ou légales, mais où la sociabilité et la débrouillardise se portent assez bien merci.

Ces résistances, jointes aux éléments soulignés précédemment, nous obligent à faire une constatation majeure : nous sommes en face d'un surprenant amalgame; si la jeunesse porte en elle des traits individualistes et conservateurs, à l'image, somme toute, du reste de la société, elle n'en manifeste pas moins une vitalité "souterraine» fort impressionnante qui nous amène à relire la réalité, à réviser nos analyses et à percevoir que cette autre chose existe bel et bien. S'impose alors à notre pensée une interprétation différente des caractéristiques présentées plus tôt.

\section{Relecture de l'individualisme}

Ainsi, ce fameux "souci de soi", de son bien-être, de sa vie présente, au premier abord signe d'un individualisme arrivé au stade "narcissique», peut aussi être perçu comme la caractéristique propre de générations nouvelles qui refusent de reporter à un hypothétique lendemain le droit de vivre mieux et de tenter d'être heureux. Comme le souligne si bien Melucci :

"L'histoire, donc la possibilité de changement, n'est pas orientée par des fins ultimes; ce qui compte, c'est l'aujourd'hui. La culture des jeunes rappelle alors la société à la valeur du présent comme unique mesure du changement, elle exige que ce qui compte s'affirme dans l'ici et maintenant. ${ }^{6}$ 
Pour ce faire, les jeunes doivent multiplier les «moyens " qui leur assurent minimalement, malgré le no future que semble leur imposer nos sociétés, de pouvoir vivre au mieux ce présent, cette vie quotidienne.

Dès lors, il n'y a plus d'individualisme. Cette quête d'un bonheur immédiat ne peut s'y enfermer. Bien que passant par soi-même, elle exige aussi, et de plus en plus, à mesure que croît l'incontrôlable quadrillage étatique, la multiplication des solidarités immédiates, à caractère plus ou moins ponctuel. Elle exige le développement d'une sociabilité qui n'a de sens et de "corps" que par la voie de rapports humains horizontaux, par une communication au ras du sol, une "communication ouverte [qui] affirme la volonté d'utiliser tous les réseaux de sociabilité qui la rendent possible ".$^{7}$

Cette communication de base, plutôt informelle, de rue, de café, d'amitié, vit et se développe malgré toutes les ressources dont dispose la communication mass-médiatique, une communication hypercodifiée, uniformisatrice au possible, telle une preuve que "l'avancée technologique $n$ 'arrive pas à gommer la puissance de la liaison $» .^{8}$

Globalement donc, un tel regard nous oblige à saisir différemment ces traits individualistes. Nous sommes amenés à comprendre que, de plus en plus, individu et communauté sont intimement liés, que l'un ne va pas sans l'autre et que le vécu actuel de la jeunesse porte en lui ces deux notions :

"[...] il existe une imbrication croissante entre les problèmes de l'identité individuelle et l'action collective; la solidarité du groupe n'est pas séparable de la recherche personnelle, des besoins affectifs et de communication des membres, dans leur existence quotidienne. " 9

\section{Relecture du conservatisme}

Une relecture identique s'impose lorsque l'on se penche sur les traits "conservateurs". Car, s'il y a, à certains égards, conservatisme, certaines de ses manifestations renvoient également à quelque chose d'autre. Ainsi, ce désir, cette quête de stabilité qui ressort des enquêtes citées plus tôt ne peut-elle pas être perçue, elle aussi, comme une évocation d'un criant besoin de communication "primaire", d'une communication possible, tangible, vérifiable quotidiennement, dans un monde où précarité, instabilité et dépersonnalisation règnent en maître ? C'est une course à la "serre chaude ", à l'intimité, à la sécurité, dans une société qui, à défaut d'éclater, nous écartèle.

À ce titre, il faut nuancer les propos d'Alberto Melucci lorsqu'il affirme que la culture des jeunes « revendique le droit au provisoire, à la 
réversibilité des choix, à la pluralité et au polycentrisme des histoires individuelles et collectives $" .10$ Melucci souligne une tendance qui pourrait se traduire par "le droit de ne plus se laisser enfermer dans des choix immuables, de se laisser, à jamais, dicter sa vie ". Cette tendance est vraie, bien que non universelle et, parallèlement, il y a aussi cette recherche d'enclaves, de havres de bien-être, de sécurité, qui peut passer, par exemple, par une nouvelle quête de la stabilité amoureuse, loin de l'éclatement et de l'expérimentation des générations des années 1960 et 1970.

Cela dit, ce n'est pas si surprenant. Au Québec, les 15-25 ans n'ont pas vécu l'embrigadement et la noirceur de l'avant-Révolution tranquille. Ils ne sont pas nés sur l'élan d'une libération à faire. Par contre, ils ont vécu dans un monde toujours plus aseptisé, où l'anonymat est force de loi. Ils ont, chemin faisant, vécu la destruction de tous ces réseaux organiques qui entouraient encore, jusqu'à l'installation complète, au début des années soixante-dix, de l'État-providence québécois, l'univers autour duquel gravitait la famille. Ils ont aussi vécu l'éclatement de cette même structure familiale. Devant cet état de fait, peut-on s'étonner de les voir rêver d'une vie plus stable qui passe, entre autres, par la relation amoureuse et par les amitiés?

Globalement donc, bien qu'il y ait dans ces manifestations des éléments troublants (par exemple : rapports gars-filles), tout ce qui est recul, par rapport aux générations des "libérations ", ne peut pas être lu exclusivement comme un retour en arrière. Il y a là, au contraire, des réflexes qui laissent entrevoir une vitalité des plus intéressantes.

\section{Refus relatif des services sociaux institutionnels}

Situons maintenant ces constats dans le cadre du débat sur la privatisation des services sociaux.

Généralement, lorsqu'on prend position dans le débat privé/ public, on le fait en fonction du futur, en fonction de ce que l'on croit être le mieux, et le plus rentable aussi, pour le système en place (ou pour tout système alternatif). On néglige parfois, cependant, la force et la présence grandissante du privé dans les faits: le privé des résistances d'une part, mais aussi, par exemple, le privé de tout l'univers des Organismes communautaires jeunesse (O.C.J.). Bref, la privatisation est déjà là, quotidiennement présente dans la vie des jeunes.

Ce n'est ni l'effet du hasard, ni la conséquence d'un machiavélique dessein des forces de droite. De nombreux jeunes, d'origines diverses, 
refusent fréquemment d'utiliser les services sociaux et préfèrent se référer à des structures plus souples, plus informelles, plus proches des "réseaux d'appartenance", parce qu'ils ne se retrouvent pas dans les multiples services institutionnels. Il y a plus d'un an, déjà, le Bureau de consultation jeunesse de Montréal (B.C.J.) lançait, à ce sujet, un cri d'alarme et soulignait :

"Si tant de jeunes refusent, même au risque d'y laisser leur santé et leur peau, l'aide sociale ou l'aide médicale, peu importe sa provenance, cela signifie concrètement que les jeunes non seulement n'y trouvent pas leur compte, mais comprennent d'instinct, ou tout simplement parce qu'ils ne sont pas si bêtes qu'on l'imagine en certains milieux, qu'ils sont au départ piégés, traqués, violentés. " 11

Piégés, traqués, violentés, voilà des mots crus, mais sans appel. Sans appel pour une minorité non négligeable, qui a passé une partie de sa vie à se faire trimbaler d'un foyer d'accueil à l'autre, avec centres d'accueil en prime et travailleurs sociaux à volonté ; sans appel aussi pour une jeunesse moins "étrennée", une jeunesse plutôt middle of the road, qui perçoit, elle aussi, que les portes closes sont nombreuses et, que dans les institutions, les avenues se font rares, avec ou sans diplôme.

Paradoxalement, tous ces jeunes ont beaucoup reçu de cet Étatprovidence. Ils sont nés et ont grandi à l'ère des grandes réformes sociales et scolaires des années 1960-début 1970, l'ère de l'assurance tous risques, qui devait leur offrir un avenir radieux. Bien sûr, la crise économique est arrivée, mais a-t-elle, à elle seule, tout gâché ? Ne fautil pas, aussi et surtout, regarder du côté de cet État maximal qui, à force de trop vouloir "protéger ", en vient à tout contrôler? Ne faut-il pas se demander si cette multiplication des interventions et des services n'a pas conduit à une extension de la normalisation et, par voie de conséquence, à l'atomisation des individus? Michel Maffesoli écrit sur cette question:

"En réalisant l'Un et l'homogénéité, l'État centralisateur promet la sécurité et il la donne, mais à quel prix ? La prise en charge totale de la vie et des passions des hommes en est le prix. L'État assurance, l'Étatprovidence entend décharger l'individu des soucis que lui donnait sans cesse l'aléa. Mais une telle domestication de l'avenir incertain, une telle planification de l'existence sociale nécessite le nivellement plus ou moins total comme condition de possibilité. Le prédateur qu'est l'homme tend ainsi à devenir un animal domestique sous le niveau égalisateur. " 12 
Si Maffesoli a raison d'insister sur l'homogénéisation de la vie sociale par un État-providence omniprésent, les éléments présentés précédemment renvoient aussi l'image d'une puissante vitalité sociétale nous obligeant à constater que, malgré tout le contrôle, il y a aussi résurgence de conduites qui défient et grugent, à leur façon, l'édifice planificateur.

Ces conduites multiples, elles se forgent et se modifient à l'image de ce social-étatique. Elles recoupent les faits énoncés plus tôt concernant la jeunesse et elle constituent, essentiellement, deux pôles : l'un, plutôt atonique et l'autre, de l'ordre de la résistance plus offensive. Parlant des effets pervers de la surprotection sociale, Maffesoli souligne que :

" La surprotection entraîne toujours soit l'atonie, soit la révolte. Il s'agit là de phénomènes complémentaires qui appartiennent tous les deux, au sens strict, à l'anomie [...] il est certain que c'est dans la logique de l'organisation étatique d'aboutir à la « massification » et ce, sur la base d'un individualisme au préalable érigé en principe. Si cette tentative échoue, du moins en partie, et toujours à terme, c'est à notre avis grâce à l'irrépressible vouloir vivre social qui ronge inlassablement les diverses formes de l'imposition mortifère. $~^{13}$

Ce " vouloir vivre " se dessine donc de manières diverses, en fonction de l'espace qui lui reste. D'un côté il passe par des conduites qui tiennent de l'atonie, renvoyant à des réflexes d'affaissement, de désengagement (ou de non-engagement), d'ennui, de repli sur soi, de déresponsabilisation. C'est un peu comme si, face à l'étouffement de la vie, il valait mieux retraiter, du moins par moments, sur son privé, sur son quotidien, sur son petit monde.

D'un autre côté, toutefois, cet attentisme (dit dans un sens non péjoratif) n'est pas la seule réponse. Devant un social-étatique qui, à tout vouloir contrôler ne contrôle plus rien, devant cette perte d'efficacité, cette impuissance chronique à répondre aux besoins et à la demande des individus, il y a aussi des réflexes plus offensifs qui, sans être exclusivement de la révolte, comme Maffesoli le dit, expriment la recherche d'une appropriation autre et de nouvelles réponses à ses besoins.

Parfois, cette offensive réside dans l'univers plutôt flou de la transgression et de la déviance sociale; parfois elle se fait plus douce, prenant la route des réseaux de nature substitutive. Dans un cas comme dans l'autre, ce sont des lieux tangibles de "socialité ", des espaces où se reconstruit et se recompose le lien social, où l'être-ensemble prime et se meut, tel le foisonnement propre à toute culture biologique. C'est à cette enseigne que logent actuellement les jeunesses du Québec. 


\section{Quelles pratiques?}

Si le "privé» a pris une telle ampleur dans la vie des jeunes d'aujourd'hui, tant sur le terrain du communautaire que dans le développement de solidarités de base, organiques - que Rosanvallon appelle des modes de régulation autogestionnaire et intro-sociale c'est qu'il représente le seul lieu possible, le seul espace disponible pour exprimer la vie, sa vie, sa créativité.

Dès lors, tout en concédant qu'il faut absolument conserver des acquis sociaux fondamentaux, tels l'universalité des services de santé, la sécurité sociale, etc., les faits parlent d'eux-mêmes et on ne peut lire la crise de l'État-providence comme étant uniquement tributaire d'une vague néo-libérale. Selon Rosanvallon, elle est aussi issue "de la décomposition ou plus exactement de la dislocation du tissu social mécaniquement [...] engendré par le dit État-providence, ce qui donne comme résultante qu'il n'y a plus assez de social entre l'État et les individus " , 14 et que, pour réouvrir cet espace, de sérieuses remises en question s'imposent. Il est donc vital de mettre de l'avant des alternatives car, comme il le dit :

«Si aucune alternative positive à l'État-providence n'est proposée par ceux qui en bénéficient le plus, nous irons vers une société bâtarde dans laquelle le renforcement des mécanismes de marché coexistera avec le maintien des formes étatisées. » 15

II est difficile de se rebiffer, de se démonter. II est absolument essentiel de proposer des avenues et des perspectives qui permettent justement de faire avancer le débat sur le renouvellement des pratiques. Pour Rosanvallon, la piste passe par ce qu'il appelle une " réduction du rôle du modèle keynésien et sa combinaison avec les modes de régulation autogestionnaire et intro-sociale $" .{ }^{16}$ Concrètement, cela pourrait se traduire par des avenues qui en viennent «à prôner à la fois moins d'État (dans les services) et plus d'État (dans les politiques) "..$^{17}$ Quoique sans garantie, les pistes sont intéressantes et visent à la fois à consolider des acquis et à établir de nouveaux droits, tout en s'efforçant de contourner ou de se sortir des méandres de l'hyper-technocratisation dans laquelle s'est enfoncé le social. Encore plus concrètement, cela passe, en définitive, par une volonté de privilégier les pratiques plus communautaires, dans l'ordre de "l'autogestion et de l'intro-social ", pour citer Rosanvallon, pratiques qui peuvent sembler " préférables aux services institutionnels lourds" "18 en ayant bien soin de "leur accorder un soutien financier effectif $" .{ }^{19}$

C'est bien évidemment sur cette voie qu'essaient de se situer la majorité des O.C.J., tel le Regroupement des maisons de jeunes. Ainsi, 
ce dernier soulignait dernièrement, devant la Commission Rochon, que ses subventions devraient être augmentées et garanties et qu'il devrait conserver une autonomie non négligeable (mais toute relative aussi) par rapport à l'infrastructure institutionnelle. Ces pratiques ne sont évidemment pas la panacée, la solution miracle. Y en a-t-il une de toute façon? Mais elles ont au moins la force d'être proches de la vie, ce qui apparaît comme un atout fondamental. Elles sont, en fait, les seules issues possibles pour sortir de l'impasse tout en en actualisant les forces.

\section{Références bibliographiques}

1 BEDARD, G. et J. GAMACHE, "Les rejetons blasés de la révolution tranquille", dans : Jeunesse : des illusions tranquilles, collectif sous la direction de M.-A. Deniger, J. Gamache et J.-F. Rene, Montréal, V.L.B., 1986, p. 192.

2 LACELle, Denyse, "Si on commençait par se regarder ", dans : Jeunesse : ..., op. cit., p. 165.

3 BAKER, Maureen, Quand je pense à demain... une étude sur les aspirations des adolescentes, Ottawa, Conseil consultatif canadien sur la situation de la femme (C.C.C.S.F.), mars 1985.

4 Gauvreau, Brigitte, "Et les jeunes, pourquoi ne se marient-ils plus ? ", La Presse, 1er mars 1986 : D-1, D-2.

5 RENE, J.-F., "Jeunesse : la résistance à l'épreuve du quotidien ", Jeunesse : ..., op. cit.

6 MelucCl, A., "Mouvements sociaux, mouvements post-politiques", Revue internationale d'action communautaire, 10/50, 1983, p. 20.

7 Ibid.

8 Maffesoli, Michel, Le Divin social, texte inédit, p. 6.

9 MelucCl, A., op. cit., p. 15.

10 Idem, p. 20.

11 BerNiER, Conrad, "Plusieurs jeunes refusent l'aide médicale ou sociale", La Presse, 27 novembre 1984 : A-10.

12 Maffesol, Michel, La Violence totalitaire, Paris, P.U.F., 1979, p. 297.

13 Maffesoli, M., "La Violence totalitaire", dans: G. Renaud, À l'ombre du rationalisme, Montréal, Albert Saint-Martin, 1984, p. 25.

14 Rosanvallon, P., La crise de l'État-providence, Paris, Seuil, 1981, p. 44. ("Points".)

15 Rosanvallon, P., op. cit., p. 136.

16 Ibid.

17 Extrait de: F. LESEMANN, Une problématique de recherche sur les services sociaux, document de travail présenté à la Commission Rochon, février 1986, p. 11.

18 Idem, p. 13.

19 Ibid. 